OPEN ACCESS

Edited by:

Carlo Perricone,

Sapienza University of Rome, Italy

Reviewed by:

Rosaria Talarico,

University of Pisa, Italy

Alessandra Bettiol,

University of Florence, Italy

*Correspondence:

Savino Sciascia

savino.sciascia@unito.it

tORCID:

Simone Baldovino orcid.org/0000-0002-4475-4117

${ }^{\ddagger}$ These authors have contributed equally to this work

Specialty section: This article was submitted to Autoimmune and Autoinflammatory Disorders, a section of the journal

Frontiers in Immunology

Received: 02 September 2021 Accepted: 04 October 2021 Published: 25 October 2021

Citation:

Arbrile M, Radin M, Rossi D, Menegatti E, Baldovino S, Sciascia S and Roccatello D (2021) Vedolizumab for the Management of Refractory Behçet's Disease: From a Case Report to New Pieces of Mosaic in a Complex Disease.

Front. Immunol. 12:769785. doi: 10.3389/fimmu.2021.769785

\section{Vedolizumab for the Management of Refractory Behçet's Disease: From a Case Report to New Pieces of Mosaic in a Complex Disease}

\author{
Marta Arbrile, Massimo Radin, Daniela Rossi, Elisa Menegatti, Simone Baldovino ${ }^{\dagger}$, \\ Savino Sciascia ${ }^{\star \ddagger}$ and Dario Roccatello ${ }^{\ddagger}$ \\ Center of Research of Immunopathology and Rare Diseases - Coordinating Center of Piemonte and Valle d'Aosta Network \\ for Rare Diseases, Department of Clinical and Biological Sciences, Complex Structure with University Management (SCDU) \\ Nephrology and Dialysis, S. Giovanni Bosco Hospital and University of Turin, Turin, Italy
}

Objectives: When treating Behçet's disease (BD), anti-tumor necrosis factor (TNF)- $\alpha$ agents have become a second-line therapy when conventional immunosuppressive drugs have failed. However, in the case of failure of treatment with anti-TNF $\alpha$ drugs, further options are limited. Based on previous reports of the efficacy of vedolizumab (VDZ) in inflammatory bowel diseases, we decided to administer VDZ to treat a patient with intestinal BD.

Methods: We present the case of a 49-year-old female patient with BD. Her clinical manifestations included erythema nodosum, oro-genital ulcers, positive Pathergy test, positive HLA-B51, and biopsy-proven intestinal BD. The patient was unsuccessfully treated with conventional immunosuppressive and several biological agents.

Results: Treatment with VDZ was started intravenously at a dose of 300 mg at 0, 2, and 6 weeks and then every 4 weeks. After the second dose of VDZ, the patient reported a marked improvement of intestinal BD and a concomitant amelioration of arthralgia, erythema nodosum lesions and aphthosis. Clinical remission was achieved at 6 months after starting VDZ.

Conclusion: VDZ might represent a valid option to treat patients with BD who are nonresponsive to standard treatments or anti-TNF $\alpha$ agents, particularly, those cases with intestinal involvement.

Keywords: vedolizumab, Behçet disease, biological drug, intestinal Behçet, biological therapy

\section{INTRODUCTION}

First identified in 1937, Behçet's disease (BD) is a multisystemic inflammatory condition often described as a part of the vasculitic spectrum, characterized by recurrent oral and genital aphthosis, skin lesions, uveitis, and, less frequently, neurologic, articular, and gastrointestinal involvement (1). BD is mostly prevalent in countries along the ancient "Silk Road", from the Mediterranean area to the far East, where it is associated with a significant prevalence of the major histocompatibility 
complex antigen HLA-B51 $(2,3)$. Before the availability of biological agents, options for the treatment of $\mathrm{BD}$ were limited to corticosteroids and conventional immunosuppressive drugs (1). The recent off-label use of biologic drugs such as infliximab and adalimumab, two anti-TNF $\alpha$ monoclonal antibodies, has improved the therapeutic armamentarium for refractory cases (4, 5). Nevertheless, not all patients fully respond to anti-TNF $\alpha$ agents, and it is quite common to experience a loss of efficacy over time in patients who initially had a beneficial effect (6).

Vedolizumab is a new biologic agent with a specific intestinal tropism, recently approved for the treatment of inflammatory bowel diseases (IBDs) $(7,8)$. VDZ binds to the $\alpha 4 \beta 7$ integrin, a glycoprotein expressed on the cell surface of circulating $B$ and $T$ lymphocytes, and blocks the interaction between the integrin and the mucosal addressin cell adhesion molecule 1 on the endothelium of intestinal blood vessels (9). The rationale for the use of VDZ in $\mathrm{BD}$ patients relies on the similar gastrointestinal involvement of the two conditions. In fact, a growing body of evidence is suggesting that IBDs and BD may be closely related and part of a common disease spectrum rather than distinct disease entities (10).

Herein we describe the case of a patient with $\mathrm{BD}$ with gastrointestinal involvement refractory and/or intolerant to several previous therapeutic approaches (including both conventional and biological disease modifying anti-rheumatic drugs, DMARDs) but who was successfully treated with vedolizumab (VDZ) and represents, to our knowledge, the first report about the use of VDZ in BD.

\section{CASE REPORT}

We present the case of a 49-year-old female patient. Her clinical history was unremarkable, aside from cutaneous psoriasis until the age of 35 when, during the post-partum period of her second pregnancy, she presented with a new onset of fever, diarrhea, and ankle arthritis. The concomitant presence of erythema nodosum and oro-genital ulcers along with the negativity for antinuclear antibodies, anti-double-stranded DNA antibodies, and normal levels of C3 and C4 supported a BD diagnosis. Colchicine and low doses of corticosteroids were started, with improvement on the aphthosis and the erythema nodosum, but with no changes in the persistence of abdominalgia and diarrhea. After a careful investigation to rule out any concomitant infectious disease, treatment with cyclosporine was started, which initially only elicited limited benefit and was subsequently suspended due to the onset of dizziness and worsening of intestinal symptoms. The ileo-colonoscopy was consistent with ileocolitis with two sigmoid colon irregularities of aphtoid aspect adjacent to macroscopically normal-appearing mucosa. The colon biopsy results reported nonspecific chronic inflammation with follicular hyperplasia of the lymphoreticular tissue with no presence of granulomas, confirming the histological diagnosis of BD.

The list of main investigations undergone by the patient is presented in Table 1. The patient attended other centers, from 2011 to 2017, where she was treated with different cDMARDs and bDMARDs as follows: sulfasalazine, which was suspended for increased liver enzymes, while azathioprine, adalimumab, infliximab, golimumab, and certolizumab were all discontinued due to lack of response. Secukinumab, introduced in 2018, was stopped regardless of the beneficial effects on BD symptoms due to the development of an anxious-depressive syndrome with suicidal thoughts, which spontaneously subsided when secukinumab was suspended. In 2019, a further attempt with golimumab was performed, with no clinical benefit and no control on the persistence of erythema nodosum, aphthosis, and diarrhea.

In November 2020, the woman was referred to our center (CMID, S. Giovanni Bosco Hub, Turin, Italy) for further evaluation. She presented with arthralgia (mainly affecting the wrists, metacarpophalangeal joints, and shoulders) and recurrent ulcerations and folliculitis on her back, forehead, and malar area. During the first evaluation, her main complaints were abdominal pain and persistent diarrhea which constantly impact her quality of life and were only partially responsive to symptomatic treatment. The physical examination showed two areas of erythema nodosum on the lower limbs (Figure 1). The Pathergy test and HLA-B51 were positive. Her laboratory profile was consistent with active $\mathrm{BD}$, characterized by markedly increased erythrocyte sedimentation rate and Creactive protein $(26 \mathrm{mg} / \mathrm{dl})$. After a multidisciplinary evaluation, taking into account the scarce efficacy of both cDMARDs and anti-TNF $\alpha$ strategies and the persistence of gastrointestinal symptoms, we decided to start treatment with

TABLE 1 | Previous investigations undergone by the patient.

Blood count $\quad$ WBC 10,000 (cells/ul); neutrophils 7,210; lymphocytes 2,470; RBC 5,700; Hb 11.3 g/dl; MCV 62 fL; PLTs 248,000

Blood tests Crea 0.74, AST 21, ALT 29, GGT 19, total protein 73, C3 1.65 g/l, C4 0.26 g/l, ESR 25, CRP 26, ANA pos 1:640, ENA neg, anti-dsDNA neg, antiphospholipid antibodies neg, HBV-DNA: neg; HCV-RNA: neg; Normal complete urine test, Uroculture: neg.

Shoulder MRI Signs of acromioclavicular fibroarthrosis, compression of the myo-tendon tract of the supraspinatus in which the tendon shows a inhomogeneous character in the pre-insertion anterior area due to tendinosis; signal alteration of the medullary bone component of the humeral shaft Unremarkable

Cerebral MR

Pelvis MRI Initial sacrum-ileitis of the right sacro-iliac joint

Abdominal echography Abdominal TC Steatotic liver disease, free of focal lesions. Outcomes of cholecystectomy. Mild hypersplenomegaly (diameter $12.5 \mathrm{~cm}$ ). Anechoic oval formation at uterine level with a diameter of $18 \mathrm{~mm}$, compatible with a follicle

appears markedly thickened, inhomogeneous, and with discrete effusion.

Colonoscopy/ Mucosal irregularities of aphtoid aspect at the sigmoid colon level. Mucosal flaps of the large intestine with mild atrophy, chronic interstitial inflammation, Colon biopsy edema, and hyperplasia of the muscularis mucosae. 

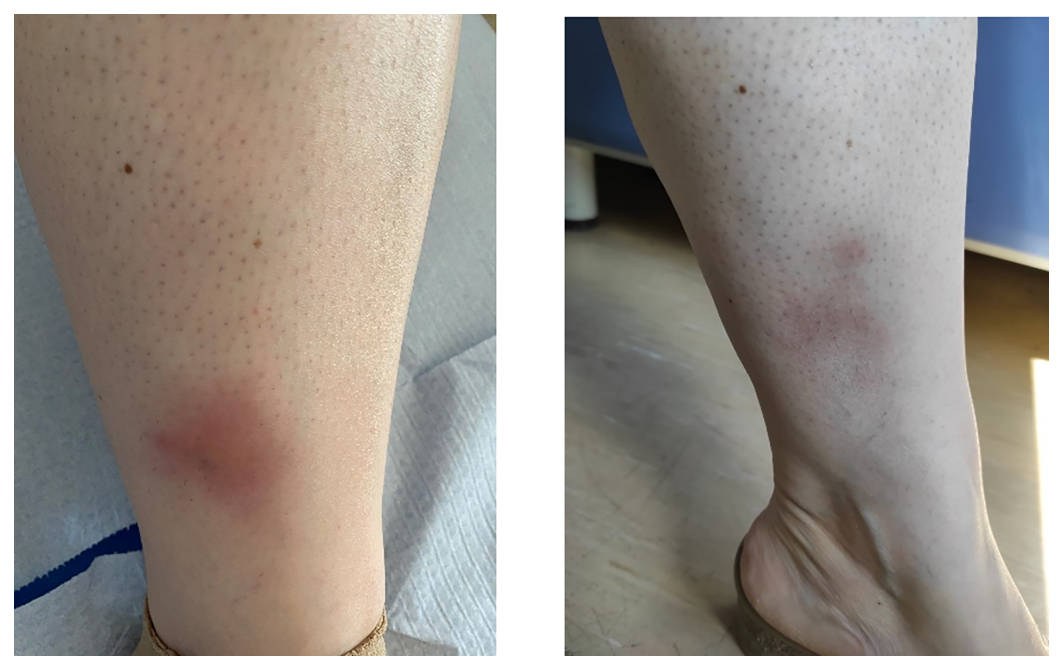

FIGURE 1 | Active tibial erythema nodosum at the first presentation of the patient, before starting with vedolizumab (left panel) and after 3 months (right panel).

VDZ, which was administered intravenously in monotherapy at a dose of $300 \mathrm{mg}$ at 0,2 , and 6 weeks and then every 4 weeks.

After the second dose of VDZ, the patient reported a marked improvement when considering gastrointestinal symptoms and arthralgia, with a decrease in the Behçet's Disease Current Activity Form 2006 (11) score from 6 (at the VDZ starting time) to 0 (after 6 months of follow-up). A significant reduction of the multiple erythema nodosum lesions affecting both legs was progressively observed, with a complete resolution after the third dose and parallel amelioration of the associated pain.

At 6 months of follow-up, no side effects were observed, and the patient did not experience further episodes of aphthosis.

\section{DISCUSSION}

In this study, we reported the case of a woman with $\mathrm{BD}$ complicated by biopsy-proven gastrointestinal involvement, who was unresponsive to conventional treatment and various anti-TNF $\alpha$ agents, and who experienced a marked resolution of the gastrointestinal symptoms when treated with VDZ.

The patient was diagnosed with BD in 2007 because of recurrent oral and genital aphthosis, erythema nodosum, arthritis, and intestinal involvement. Since BD diagnosis is not characterized by specific laboratory, radiologic, or histologic findings, the diagnosis of this condition relies mainly on clinical evaluation (1). The most recent classification criteria are the International Criteria for Behçet Disease, published in 2006 and revised in 2014, which require a score of 4 from the following list: oral aphthosis, genital aphthosis, ocular manifestations, skin involvement, vascular manifestations, neurologic manifestation, and positive Pathergy test (12). Gastrointestinal involvement in $\mathrm{BD}$ remains infrequent. Interestingly, geographical differences among BD patients have been reported, with colon involvement being more frequent in patients from Europe and North America, while ileocecal involvement appears to be more common in Japan (13).

The aim of the treatment in $\mathrm{BD}$ is maintaining remission and improving the quality of life of the patient, but new therapeutic approaches are still needed. Figure 2 illustrates the current therapeutic options for BD treatment and their main application related to clinical symptoms.

For many years, the only bDMARDs available for the management of BD were anti-TNF $\alpha$ agents. More recently, anti-cytokine-related (e.g., IL-6) strategies have been suggested as therapeutic options for $\mathrm{BD}$, with a good safety and efficacy profile $(14,15)$. Recently approved for IBDs, VDZ, targeting a completely novel mechanism of action, could represent a new tool in the therapeutic armamentarium of BD specialists. VDZ can be considered as an "integrin antagonist" due to its ability of binding to the $\alpha 4 \beta 7$ integrin (a molecule identifiable on Tlymphocytes and mononuclear cells). This gastrointestinalspecific interaction reduces the side effects associated with systemic immunosuppression. The severe intestinal involvement of the patient discussed in our study was the main reason for switching to VDZ from anti-TNF $\alpha$ agents. Despite the specific gastrointestinal effect, in several studies VDZ has proved to be effective also when managing the extraintestinal manifestations of IBDs (i.e., skin involvement, uveitis, and arthritis), even if the mechanism remains unknown to this day $(16,17)$. These observations are in line with our case, as during follow-up, after 6 months of treatment with VDZ, our patient remained asymptomatic and without any sign of active inflammation. Of note is that no side effects were reported.

Meant to prevent the migration of inflammatory mononuclear cells into the inflamed mucosa in patients with IBD, VDZ could also mitigate the inflammatory process in $\mathrm{BD}$ (3), limiting the interaction of lymphocyte integrins (as expressed by CD4+ and CD8+ naive T cells and CD4+ and CD8+ memory T cells) and their endothelial ligands (18). 


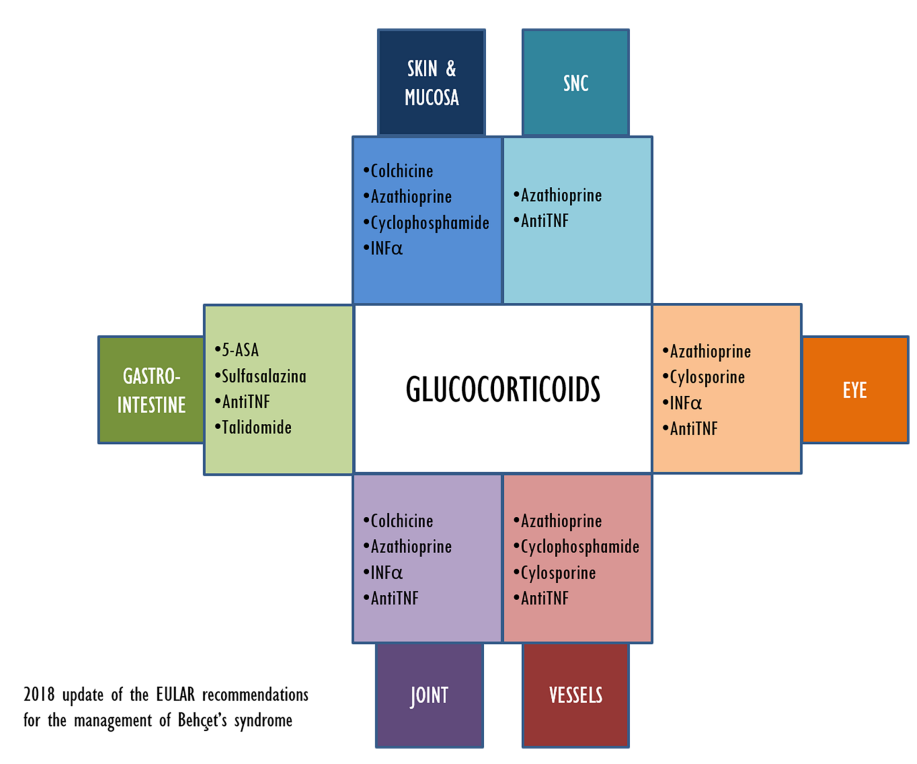

FIGURE 2 | 2018 update of the EULAR recommendations for the management of Beçhet's syndrome.

\section{CONCLUSION}

In conclusion, this case shows the efficacy of VDZ in inducing remission at 6 months of follow-up in a patient not responsive to anti-TNF $\alpha$ agents. When treating BD, the anti-TNF $\alpha$ agents have become a standard when conventional immunosuppressive drugs have failed. However, in case of anti-TNF $\alpha$ agents failure, the options are limited. A switch to another anti-TNF $\alpha$ drug is a current possibility, but in our case it was not effective. Based on previous reports of its efficacy in IBDs, we decided to administer VDZ to treat the intestinal involvement of our patient. This led not only to a satisfactory gastrointestinal response but also to the concomitant disappearing of ulcerations, arthralgia, and a reversion of the skin lesions.

Some limitations should be acknowledged, mainly the short follow-up which is currently up to 6 months. Further larger cohort studies with a longer follow-up are needed to provide more evidence on these preliminary findings, especially in regard to the dosage of VDZ, the duration of treatment, the compatibility with other immunosuppressive agents, and the efficacy on patients without intestinal involvement. However, VDZ might represent a valid option for the treatment of patients with $\mathrm{BD}$ who are not responsive to standard treatment or anti-TNF agents.

\section{REFERENCES}

1. Akdeniz N, Elmas ÖF, Karadağ AS. Behçet Syndrome: A Great Imitator. Clin Dermatol (2019) 37(3):227-39. doi: 10.1016/j.clindermatol.2019.01.001

2. De Menthon M, LaValley MP, Maldini C, Guillevin L, Mahr A. HLA-B51/B5 and the Risk of Behçet's Disease: A Systematic Review and Meta-Analysis of

\section{DATA AVAILABILITY STATEMENT}

The raw data supporting the conclusions of this article will be made available by the authors, without undue reservation.

\section{ETHICS STATEMENT}

Ethical approval was not provided for this study on human participants because the study is a case report. Written consensus was retrieved by the patient and the research was run in accordance with the declaration of Helsinki. The patients/ participants provided their written informed consent to participate in this study.

\section{AUTHOR CONTRIBUTIONS}

MA participated in data collection, interpretation of results, and drafting of the manuscript. MR, DRos, EM, SB, SS, and DRoc participated in data collection, interpretation of results, manuscript preparation, and final review. All authors contributed to the article and approved the submitted version.
Case-Control Genetic Association Studies. Arthritis Care Res (2009) 61 (10):1287-96. doi: 10.1002/art.24642

3. Leccese P, Alpsoy E. Behçet's Disease: An Overview of Etiopathogenesis. Front Immunol (2019) 10:1067(MAY). doi: 10.3389/fimmu.2019.01067

4. Esatoglu SN, Hatemi G. Update on the Treatment of Behçet's Syndrome. Intern Emerg Med (2019) 14(5):661-75. doi: 10.1007/s11739-019-02035-1 
5. Alpsoy E, Leccese P, Emmi G, Ohno S. Treatment of Behçet's Disease: An Algorithmic Multidisciplinary Approach. Front Med (2021) 8:624795. doi: $10.3389 /$ fmed.2021.624795

6. Caso F, Costa L, Rigante D, Lucherini OM, Caso P, Bascherini V, et al. Biological Treatments in Behçet's Disease: Beyond Anti-TNF Therapy. Mediators Inflamm (2014) 2014:107421. doi: 10.1155/2014/107421

7. Balzola F, Cullen G, Ho GT, Russell R. Vedolizumab as Induction and Maintenance Therapy for Crohn's Disease: Commentary. Inflammation Bowel Dis Monit (2014) 14(2):55-6. doi: 10.1056/NEJMoa1215739

8. Feagan BG, Rutgeerts P, Sands BE, Hanauer S, Colombel JF, Sandborn WJ, et al. Vedolizumab as Induction and Maintenance Therapy for Ulcerative Colitis. N Engl J Med (2013) 369(8):699-710. doi: 10.1056/ nejmoa1215734

9. Wyant T, Fedyk E, Abhyankar B. An Overview of the Mechanism of Action of the Monoclonal Antibody Vedolizumab. J Crohn's Colitis (2016) 10 (12):1437-44. doi: 10.1093/ecco-jcc/jjw092

10. Kim ES, Chung WC, Lee KM, Lee BI, Choi H, Han SW, et al. A Case of Intestinal Behcet's Disease Similar to Crohn's Colitis. J Korean Med Sci (2007) 22(5):918-22. doi: 10.3346/jkms.2007.22.5.918

11. C HJ, S MR, R HJ, B HJ. Cross-Cultural Adaptation and Validation of the Behcet's Disease Current Activity Form in Korea. Korean J Intern Med (2015) 30(5):714-8. doi: 10.3904/KJIM.2015.30.5.714

12. Davatchi F, Assaad-Khalil S, Calamia KT, Crook JE, Sadeghi-Abdollahi B, Schirmer M, et al. The International Criteria for Behçet's Disease (ICBD): A Collaborative Study of 27 Countries on the Sensitivity and Specificity of the New Criteria. J Eur Acad Dermatol Venereol (2014) 28(3):338-47. doi: $10.1111 /$ jdv.12107

13. Bayraktar Y, Özaslan E, Van Thiel DH. Gastrointestinal Manifestations of Behcet's Disease. J Clin Gastroenterol (2000) 30(2):144-54. doi: 10.1097/ 00004836-200003000-00006

14. Akiyama M, Kaneko Y, Takeuchi T. Effectiveness of Tocilizumab in Behcet's Disease: A Systematic Literature Review. Semin Arthritis Rheumatol (2020) 50 (4):797-804. doi: 10.1016/j.semarthrit.2020.05.017
15. Atienza-Mateo B, Beltrán E, Hernández-Garfella $M$, Valls Pascual E, Martínez-Costa L, Atanes A, et al. Tocilizumab in Behçet's Disease With Refractory Ocular and/or Neurological Involvement: Response According to Different Clinical Phenotypes. Clin Exp Rheumatol (2021) 132(5):37-42.

16. Fleisher M, Marsal J, Lee SD, Frado LE, Parian A, Korelitz BI, et al. Effects of Vedolizumab Therapy on Extraintestinal Manifestations in Inflammatory Bowel Disease. Dig Dis Sci (2018) 63(4):825-33. doi: 10.1007/s10620-0184971-1

17. Hanzel J, Ma C, Casteele NV, Khanna R, Jairath V, Feagan BG. Vedolizumab and Extraintestinal Manifestations in Inflammatory Bowel Disease. Drugs (2021) 81(3):333-47. doi: 10.1007/s40265-020-01460-3

18. Mitroulis I, Alexaki VI, Kourtzelis I, Ziogas A, Hajishengallis G, Chavakis T. Leukocyte Integrins: Role in Leukocyte Recruitment and as Therapeutic Targets in Inflammatory Disease. Pharmacol Ther (2015) 147:123-35. doi: 10.1016/j.pharmthera.2014.11.008

Conflict of Interest: The authors declare that the research was conducted in the absence of any commercial or financial relationships that could be construed as a potential conflict of interest.

Publisher's Note: All claims expressed in this article are solely those of the authors and do not necessarily represent those of their affiliated organizations, or those of the publisher, the editors and the reviewers. Any product that may be evaluated in this article, or claim that may be made by its manufacturer, is not guaranteed or endorsed by the publisher.

Copyright (c) 2021 Arbrile, Radin, Rossi, Menegatti, Baldovino, Sciascia and Roccatello. This is an open-access article distributed under the terms of the Creative Commons Attribution License (CC BY). The use, distribution or reproduction in other forums is permitted, provided the original author(s) and the copyright owner(s) are credited and that the original publication in this journal is cited, in accordance with accepted academic practice. No use, distribution or reproduction is permitted which does not comply with these terms. 